\title{
Impact of DBTMA (Dual Busy-Tone Multiple Access) on AODV (Ad-Hoc on Demand Vector) Routing Protocol
}

\author{
S. V. Vanitha, G. Mohankumar \\ ${ }^{1}$ ECE Department, Park College of Engineering and Technology, Coimbatore, India \\ ${ }^{2}$ Mechanical Department, Park College of Engineering and Technology, Coimbatore, India \\ Email: svvanitha_2005@yahoo.co.in
}

How to cite this paper: Vanitha, S.V. and Mohankumar, G. (2016) Impact of DBTMA (Dual Busy-Tone Multiple Access) on AODV (Ad-Hoc on Demand Vector) Routing Protocol. Circuits and Systems, 7, 3604-3616. http://dx.doi.org/10.4236/cs.2016.711305

Received: May 3, 2016

Accepted: May 10, 2016

Published: September 8, 2016

Copyright $\odot 2016$ by authors and Scientific Research Publishing Inc. This work is licensed under the Creative Commons Attribution International License (CC BY 4.0).

http://creativecommons.org/licenses/by/4.0/

\begin{abstract}
The multi-hop wireless networks that provide the feasible means of communication and information access in real time services are named as Mobile Ad-hoc Networks (MANETS). The Dual Busy-Tone Multiple Access (DBTMA) mechanism concedes the RTS-CTS scheme to establish communication between two nodes and medium access for applications with a high QoS requirement by assigning two narrow band busy-tones to notify the on-going transmission. In this paper, we obtained results relative to the interest of AODV based reactive routing protocol for MANETS and DBTMA mechanism. The performance is governed under real time sound traffic through simulation using NS-2. The performance of the protocol is measured in terms of various QoS metrics that include route discovery time, throughput, delay and hops per route which are calculated, and graphs have been plotted. A simulation result shows that very substantial improvements in terms of AODV performance parameters and minimum delay are attained due to increased routing responsiveness.
\end{abstract}

\section{Keywords}

802.11e, AODV, MAC Protocols, Quality of Service, DBTMA

\section{Introduction}

An ad-hoc network is a collection of wireless hosts also named as Data-D-Hoc networks without an infrastructure. The ad-hoc network is a temporary network, which deploys the applications like battlefield communication, disaster recovery, etc. The network does not rely on an established infrastructure or on a central control. The adhoc networks that are autonomous or multi-hop wireless extension to the internet are 
called Mobile Ad-Hoc Networks (MANETS).

Routing is the process of selecting a path to the destination to send the data traffic. Routing Protocols are of two classifications: Proactive Routing protocol and Reactive Routing. In proactive routing, table-driven method is used in which each node of the network will update to the network. A periodic update is made in the routing table and the information is transmitted throughout the network to maintain its consistency. Hence, the delay in reaching the destination is reduced. However, the protocol needs more resources for the updating of information in the table. The proactive routing protocols are like DSDV (Destination Sequenced Distance Vector), Wireless Routing Protocol (WRP), and Global State Routing (GSR). In Reactive Routing Protocols, it is initiated only when there is a demand for route by a node having information to transmit. For this, a node in the network has to initialize the route discovery. The route discovered is maintained by route maintenance mechanism until the destination becomes un-accessible. The nodes that act as routers are meant for maintaining the routes for active destinations. The reactive routing can reduce the communication overhead and also reduces the delay; ex: Ad-Hoc On-Demand Vector, Dynamic Source Routing (DSR) [1], Temporarily Ordered Routing Algorithm (TORA) [1], Location Aided Routing (LAR) paper. Margins, column widths, line spacing, and type styles are built-in; examples of the type styles are provided throughout this document and are identified in italic type, within parentheses, following the example. Some components, such as multi-leveled equations, graphics, and tables are not prescribed, although the various table text styles are provided. The formatter will need to create these components, incorporating the applicable criteria that follow.

\section{Ad-Hoc On-Demand Vector (AODV)}

The routing in Ad-Hoc networks needs to be dynamic and multi-hop. The adaptive routing method for Ad-Hoc networks is AODV. The channel access procedure of AODV is shown in Figure 1. In a timely manner, the response of AODV towards link breakages and topology changes makes it suitable for those networks. The AODV has a distinguishing feature of using a destination sequence number that is created from the destination nodes. The AODV ensures loop-free routes using the destination sequence number. AODV uses the broadcast route discovery method and uni cast route reply message. The route discovery of AODV is larger as it is network wide flooding, but it

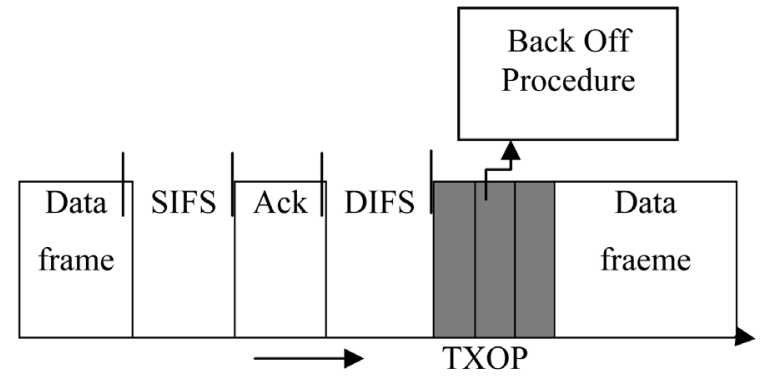

Figure 1. Channel access procedures of AODV. 
does not need global periodic routing advertisements. As the number of route discoveries is more, the routing load of AODV is large (in the 50 node case, as many from 6.5 routing packets per data packet, and 2 routing bytes per data byte). The delay characteristic of AODV protocol [1] is found poor, because of the source routing property. In the source routing protocols, the delay is longer as the route discovery makes long time as every intermediate node will try to extract the information before it is forwarded. [2] Throughput (Packet Delivery Fraction) of AODV is lower because of higher packet drop rates. Throughput increases with decrease in mobility. The On-Demand protocols will drop packets during route discovery, when a route expires, a new route must be found. The AODV protocol uses dynamic routing in order to deliver packets to any destination in an ad-hoc mode.

The following presumptions are made in an AODV Protocol [1]:

- Deciding the placement of static nodes.

- RTS/CTS control packets disabled.

- Fragmentation of frame size is disabled.

- Two-ray way propagation path loss and signal model is considered.

- Traffic/application types are configured for AC-VO is Audio, for AC-VI, V is MPEG4, for AC-BE is CBR, for AC-BK is FTP.

\section{Medium Access Control (MAC) Protocols Basic}

The MAC protocols are introduced for the nodes to access the channel with reduced collision [3]. The Ad-Hoc network MAC protocols [4] are basically classified into three, namely. (i) Contention based protocol scheme. (ii) Contention based protocols with reservation mechanism. (iii) Contention based protocols with scheduling mechanism. A contention based protocols are divided into two categories. (1) Sender initiated (SI-MAC) Protocols. (2) Receiver initiated (RI-MAC) Protocols [4] [5]. The Sender initiated protocol is meant for Single channel protocol and Multi-channel protocols. Under single channel protocol the MACAW (Medium Channel Access for Ad-hoc Wireless) and FAMA (Frequency Acquisition Multiple Access). Under multi-channel Access BTMA (Busy-Tone Multiple Access), DBTMA (Dual Busy-Tone Multiple Access) are available [6]. The MAC protocols or channel access schemes has an impact on the routing protocols to improve the performance in terms of throughput, delay, number of hops per route etc.

\section{Analysis of the DCF and PCF}

The Carrier Sense Multiple Access with typical Collision Avoidance (CSMA/CA). Carrier sensing is performed through physical and also virtual mechanisms.DCF is the Distributed Co-ordination Function that makes the basis for CSMA/CD. The medium is sensed by the station to determine if some other station is beaming in the physical mechanism. Distributing the reservation information with RTS/CTS exchanges in MAC is called as virtual carrier sensing. If the channel is found busy, the station waits for a DIFS (DCF Inter-frame Spacing) duration [7]. After this inter-frame space, the 
station transmits the DATA frame. If the medium is sensed busy during the DIFS time interval, then back-off procedure is enabled. The back off is enabled even if the channel is found busy when sensing for the foremost time. The backlog is invoked, the station waits when transmission is over. At the end of the current transmission, the station waits for a DIFS. The medium was found to be jobless for a DIFS in some conditions and [5], the station performs an additional backup wait before original transmission. [8]. The back-off counter and timer is put to some arbitrarily selected value. If the medium is idle throughout the back-off interval and the counter expires, the frame is transmitted. If the medium becomes busy during this interval, the timer is frozen at its present value but not reset. The station waits for the medium to become free, waits for a DIFS, and does the backup wait again, melting off the timer. This process goes on till the back-off timer expires and the station starts its transmission [6].

Point Coordination Function (PCF) is a premium, polling-based access mechanism which involves the presence of an AP (Access Point) that acts as Point Coordinator (PC). In the PCF mode, time is divided into super-frames. Each super-frame consists of a contention period where DCF uses a contention-free period (CFP). This protocol does not provide service differentiation and prioritization.

\section{DBTMA (Dual Busy-Tone Multiple Access)}

DBTMA is the extension of BTMA [6] (Busy-Tone Multiple Access) scheme in which the transmission channel is divided into two:

-a Data channel for data transmission

-a Control channel-to transmit the control signals along with Busy tones

When a node is ready for transmission it senses the channel for busy tone active. If not, it turns ON the busy tone signal and starts transmission. Otherwise, the packet for transmission is rescheduled after some random delay. Any other node that senses the carrier signal on the incoming data channel also transmits the busy tone signal on the control channel [6], thus preventing those two neighboring nodes from transmitting at the same time. In DBTMA, when a node has data to transmit, it will send a RTS (Request To Send) signal to the channel. The destination or the receiver that has to receive the data will reply, CTS (Clear To Send) signal along with BTr (Receive Busy Tone). On hearing the CTS from the hardware of the node transfer, the sender will raise the $\mathrm{BTt}$ signal high and starts the data transmission [8]. The BTt (transmit Busy Tone) and BTr (receive Busy Tone) are placed on the control channel that is separated with spectral separation is shown in the Figure 2.

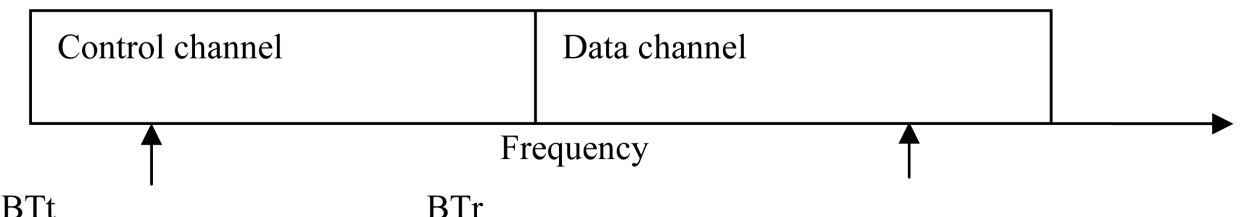

BTt

$\mathrm{BTr}$

Figure 2. DBTMA scheme. 
All the nodes sensing the BTt signal will determine that they cannot receive data. All other nodes will defer from transmitting on sensing BTr signal. This mechanism creates the hidden terminals to back-off and exposed terminals to use the channel. The transmitter produces the transmits the busy tone BTt and is done to protect the RTS packets. Another busy tone BTr, is put up by the receiver, acknowledges the RTS packet and provides uninterrupted protection for the incoming data packets. The RTS packet, and the receive busy tone make the chance of successful RTS reception. The DBTMA protocol is the fundamental on the packet and twice narrow-bandwidth, out-of band busy tones are implemented with enough spectral separation on the unicast shared channel. $\mathrm{BTt}$ (the transmit busy tone) and $\mathrm{B} \operatorname{Tr}$ (the receive busy tone), shown whether the node is forwarding RTS packets or receiving packet transfer data, respectively. The transmits the busy tone (BTt) provides security for the RTS packets to increase the chance of successful RTS reception at the designated receiver. All nodes sensing any busy tone are not admitted to send RTS requests. When the start of the better signal is felt, a node forward and transfers the RTS packet is required to abort such transmission immediately (8).

Figure 3 shows the fundamental finite state machine of DBTMA and it explains, when a system has a data packet to send while it is in the IDLE state, it tries to sense the BTt and the BTr busy tone signals. If none of the busy signals are present (which signifies that no one in node A's transmission area is receiving data packet or sending RTS packets), it works on its busy signal, sends an RTS packet to node B, and goes into the S-RTS (Send-RTS) state. Node A turns off its busy signal, sets a timer, and extends into the WF-BTR (Wait For-BTR) state. When node B receives the RTS packet, it hoist its busy signal BTr, replying to node A and announcing that it is awaiting for the incoming data packet. And so it puts up a timer and runs into the WF-DATA state.

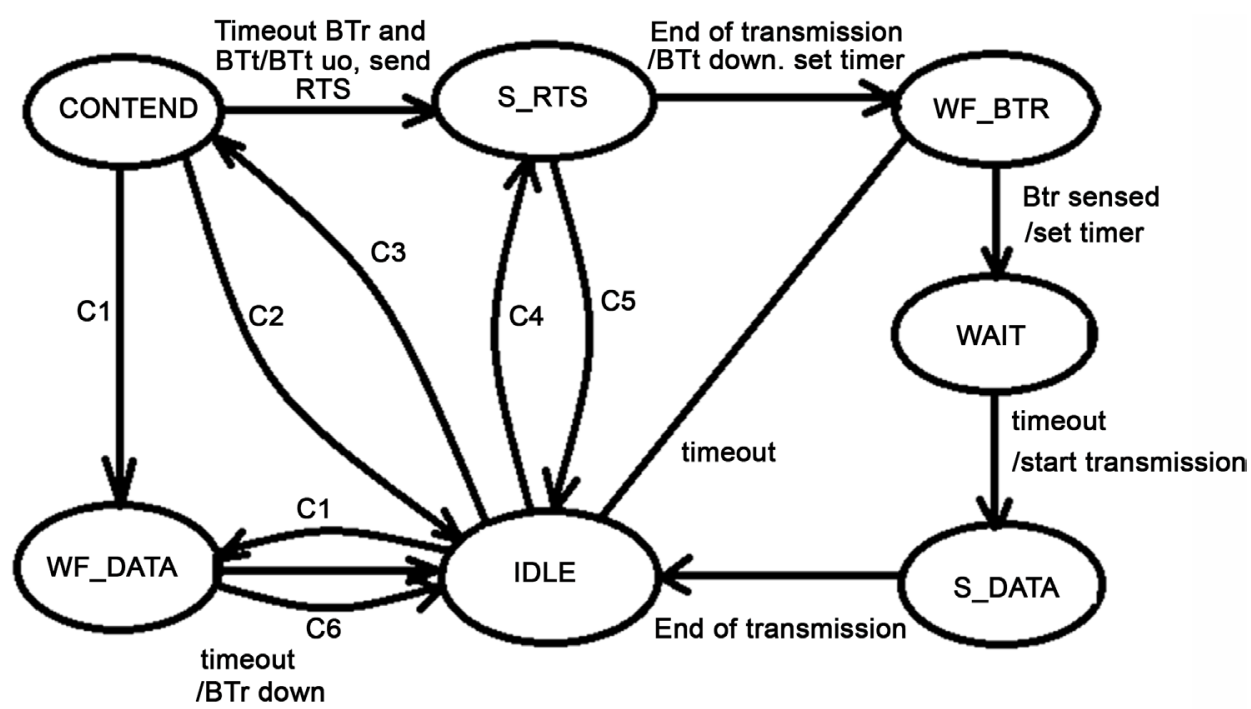

Figure 3. Fundamental finite state machine of DBTMA with BTt. C1: RTS/BTr up, set Timer; C2: timeout, BTr/BTt; C3: ready, BTr/Btt/set timer; C4: ready, BTr and BTt/BTt up, send RTS; C5: sense BTr/BTt down, abort Transmission; C6: data received/BTr down. 
Node A continuously monitors the busy signal when it is in the WF-BTR state. When a signal is felt, it recognizes that its channel request has been verified and reached. Before node A sends the data packet, it waits a mandatory waiting time (twm $=2 \mathrm{~T}$ ) in the WAIT state. This mandatory waiting time is intended to permit all possible RTS transmissions in the orbit of the recipient to be aborted. Upon timeout in the WAIT state, node A into the S-DATA (Send-DATA) state and transports the information package. At the end of the its transmission, node A goes into the IDLE state. Based on the successful reception of the data packet, node B turns off the BTr signal and passes into the IDLE state, ending the communication. If node $B$ does not receive the data packet before the timer expires, if will off the BTr signal passes into the IDLE state.

Upon timeout in the CONTEND state, node A turns its busy signal and sends its RTS packet if no busy tone signal is felt. Differently, it moves backwards into the IDLE state. From the position of the other nodes in the region, their operations can be identified as follows:

When the BTt and/or the better signal is felt, a node is not permitted to send any RTS request. When the beginning of a better signal is sensed while a client is in the S-RTS state, it aborts its RTS transmission, turns off its better signal, and moves back to the IDLE state. Figure 4 shows the timing of Node A and Node B while it responds to busy Tone signals.

The following are the assumptions for DBTMA protocol:

- The wireless transmission [4] range of the ad hoc network in which the DBTMA scheme functions is on the order of tens to hundred of meters [2]. In that location is no capture effect or fading on the channel.

- The packet is not clearly observed by the receiver, when there is any overlap of transmission at the receiver side. Packet errors are caused by packet collisions.

- The data information processing time is obtained.

- The data signal and the busy tone signal have the unique transmission range.

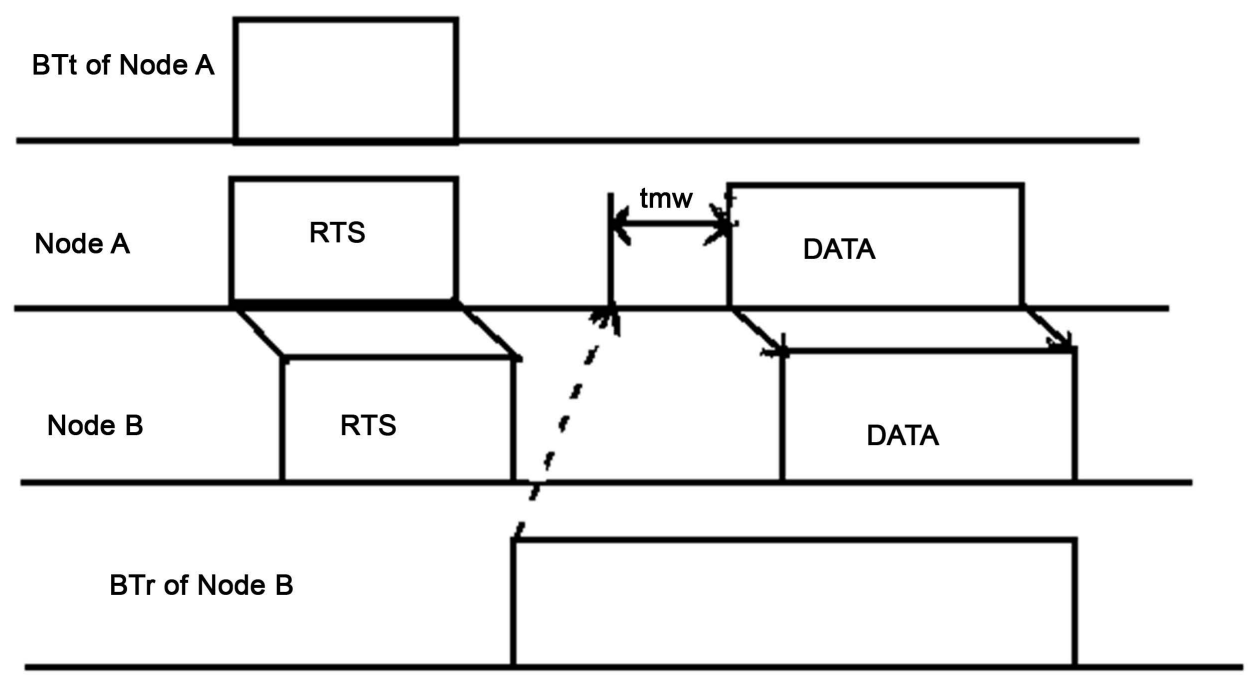

Figure 4. Timing performance diagram of DBTMA with transmission. 
- The noise between the busy tone signals and the data signal is negligible. The bandwidth consumption of the data signal is more when compared to busy tone signal that is negligible.

- The transmission time of data, the transmission time of RTS packet, and the one way propagation delay has its maximum value as $\delta, \gamma$ and $\tau$ respectively.

- The busy tone detection delay is connected, which depends on the communication hardware and might not, in general, be negligible.

- The compulsory (mandatory) waiting time is set to twm $=2 \mathrm{~T}$.

- The transmission time of the RTS packet is larger than $\mathrm{td}+4 \mathrm{~T}$.

- The large number of nodes in a network generates a poisson traffic in, which mean aggregate rate is calculated as $\lambda$ channel requests per second.

\section{Communication Rules of DBTMA}

The DBTMA communication rules are mentioned below:

- Initialization is made by powering up, a node goes into the IDLE state. We accept that both the transmitter (A) And the Receiver (B) are in the IDLE coefficient and non idle state before the CONTEND state.

- RTS control packet transmission: When source A has a data packet for transmission to the destination B, it tries to sense the busy tones, BTr and the BTt signals. The busy-tone signal BTt is raised when no busy tone is available and it sends an RTS packet to B, and gets into the S_RTS state. If source node A senses a busy tone signal, it triggers a random timer (chosen from $[0, \mathrm{BI}]$ ) and moves into the CONTEND state.

- Wait for $\mathrm{BT}_{\mathrm{r}}$ signal: When RTS transmission is completed, $\mathrm{BT}_{\mathrm{t}}$ signal is turned off by station $\mathrm{A}$ and it sets a timer to $(\mathrm{td}+2 \mathrm{~T})$ second, and moves into the WF_BTR state.

- Wait time for data packet: When destination $B$ receives the RTS packet from source $\mathrm{A}$, it raises its $\mathrm{B} T r$ signal and sets a timer to $(\delta+\mathrm{td}+2 \mathrm{~T})$ second, and moves into the WF_DATA state.

- Compulsory wait time: When A senses a better signal in the WF_BTR state, it sets a timer to $(\mathrm{t} \omega \mathrm{m}=2 \mathrm{~T})$ second and extends into the WAIT state.

- Data transmission to S_DATA: When there is timeout in the WAIT state, node A transmits the data packet to destination and passes into the S_DATA state.

- End of data transmission: At the end of the DATA transmission from A to B, node A goes into the IDLE state.

- Receive data packet at the destination: When the information packet is received or timeout happens in the WF_DATA state, B puts off the BTr signal and moves into the IDLE state.

- CONTEND stage-Timeout: When there is timeout in the CONTEND state, node $A$ tries to sense the $B T_{r}$ and the $B T_{t}$ signals again. If no busy tone signal is sensed at the medium, it works on its busy signal and sends an RTS packet to B, and exits into the S_RTS state. If node A senses a busy tone signal BTr it moves backwards into the IDLE state. 
- Timeout of back-off Timer: The timeout condition in the WF_BTR state, makes node A to move into the IDLE state.

\section{Route Discovery Time}

When a node is ready with data and needs to determine the route to its destination, it sends a RREQ (Route ReQuest) message through flooding. Thus the route request is broadcast to their neighbors. The intermediate node will have the details on a reverse route to the originator. When the destination realizes that itself as the requested node, it gives the reply as RREP (Route Reply) message along the reverse path. The intermediate nodes through which RREP message travels will update the routing table with new route information.

Each node in a network maintains a route request buffer that contains a list of recently broadcasted route requests. The route request buffer consists of a pair of values in each entry as the address of the node originated the request, and the route request identification number (RREQ ID) [7]. This RREQ ID uniquely identifies the request originated by a given node. The data packets will be dropped at high load traffic in the buffer. During the route discovery session the data packet drop is more in On-Demand routing protocols. The data acquisition time is directly proportional to the distance between the source and destination.

Route discovery time $\tau \infty \mathrm{D}_{\text {sd }}$

Table 1 shows the time values of route discovery Time in AODV and DBTMA protocols and Figure 5 shows the route discovery time plot of AODV and DBTMA. Route expiry is the concept used in AODV which implies it will drop some of the packets when a route expires and a new route must be found.

Table 1. Route discovery time in AODV and DBTMA.

\begin{tabular}{ccc}
\hline & \multicolumn{2}{c}{ Time (ms) } \\
\cline { 2 - 3 } Number of nodes & AODV & DBTMA \\
\hline 165 & 0.92 & 0.85 \\
545 & 0.81 & 0.77 \\
705 & 0.77 & 0.68 \\
885 & 0.63 & 0.55 \\
1065 & 0.52 & 0.45 \\
1245 & 0.46 & 0.38 \\
1425 & 0.38 & 0.29 \\
1605 & 0.22 & 0.19 \\
1785 & 0.18 & 0.08 \\
\hline
\end{tabular}




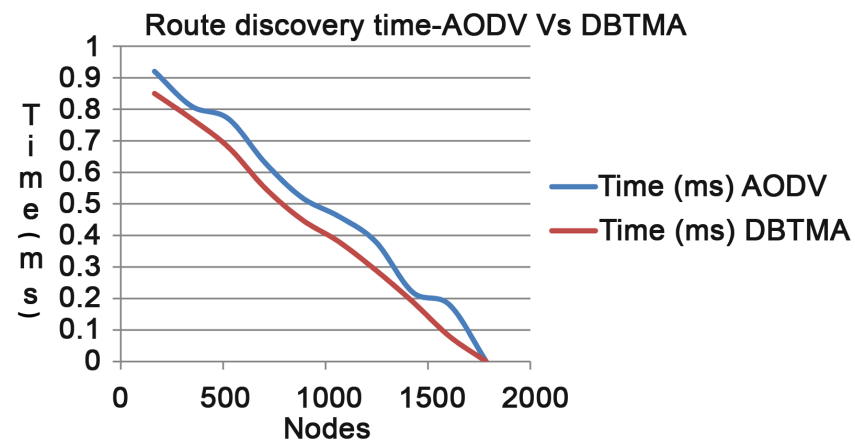

Figure 5. Route discovery time of AODV vs DBTMA.

\section{Number of Hops per Route}

When a node requires a route to destination, the route discovery process is initiated within the network. Once a route or all the possible routes are discovered the route discovery process will be completed. Depending upon the number of hops per route, the shortest path will be selected and the route establishment is made. The information about route discovery is updated in the routing table of all intermediate nodes that helps in finding the number of hops per route. This hop-by-hop method of routing is based on the fact that different nodes of the network will have different views on the topology. Hop count is used to calculate the number of hops required by a node to reach the destination. This is explained using the Figure 6. Calculation of cost (number of hops required to reach the destination) per route is done on the routing table (Table 2) of each node. Using the route method, based on:

Node A can be reached using certain hops and the establishment of route is based on the hop count. The value on the link indicates the hop or cost values and this generates higher packet drops and collision rate probability.

The number of hops per route (for 50 Nodes) is shown in Table 3 and Figure 7 for AODV and DBTMA respectively.

\section{Delay}

Average end-end delay calculation is the sum or amount of time taken by a packet to go from source to destination and it is used to find delays caused during route discovery latency, queuing at the interface queue, vice-verse transmission time and delays are considered at the hybrid MAC layer, propagation delays and transfer times. Delay in AODV protocol is comparatively more and therefore the MAC protocol that is combined with AODV can improve its performance. The Table 4 and Figure 8 shows the delay comparison of DBTMA (MAC protocol) and AODV routing protocol.

\section{Throughput}

The throughput of an AODV routing protocol can be calculated as the ratio between total number of data packets sent by the sender to the total number of data packets received at the receiver. The throughput of AODV and DBTMA can be compared using the Table 5. The Figure 9 shows the throughput simulations of AODV and DBTMA. 


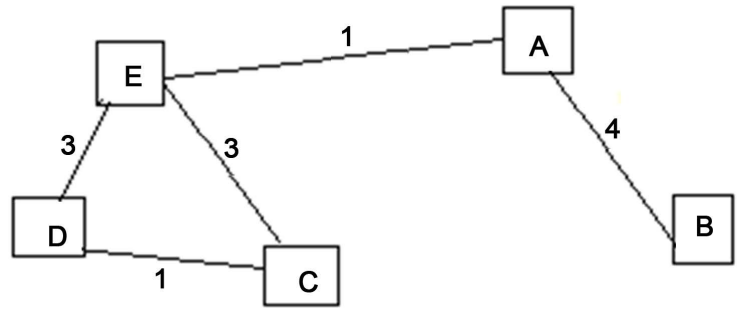

Figure 6. Number of hops per route to reach A.

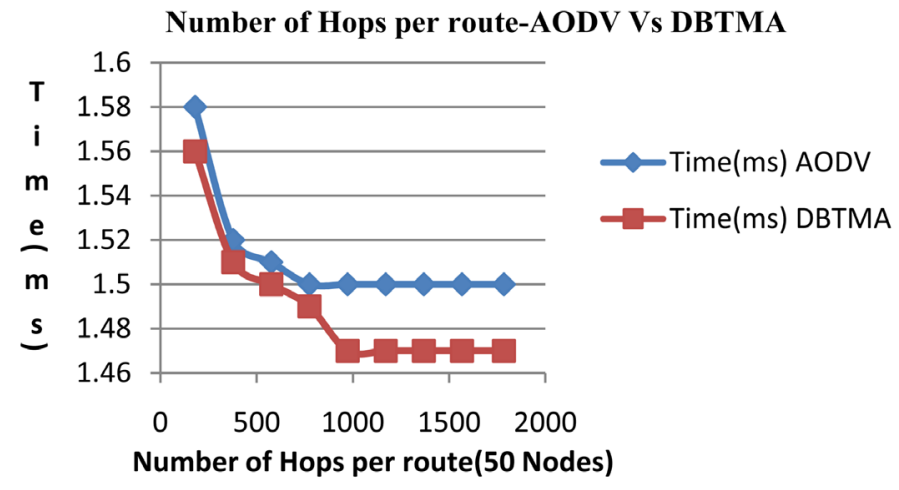

Figure 7. Hops per route-AODV vs DBTMA.

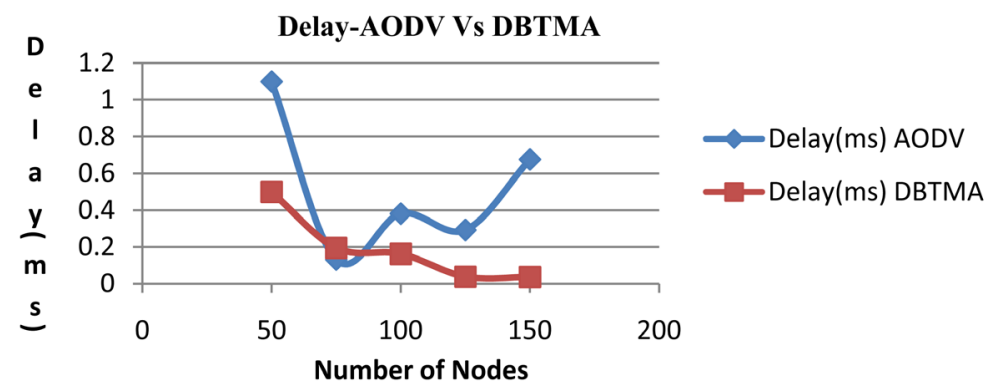

Figure 8. Average end-end delay-AODV vs DBTMA.

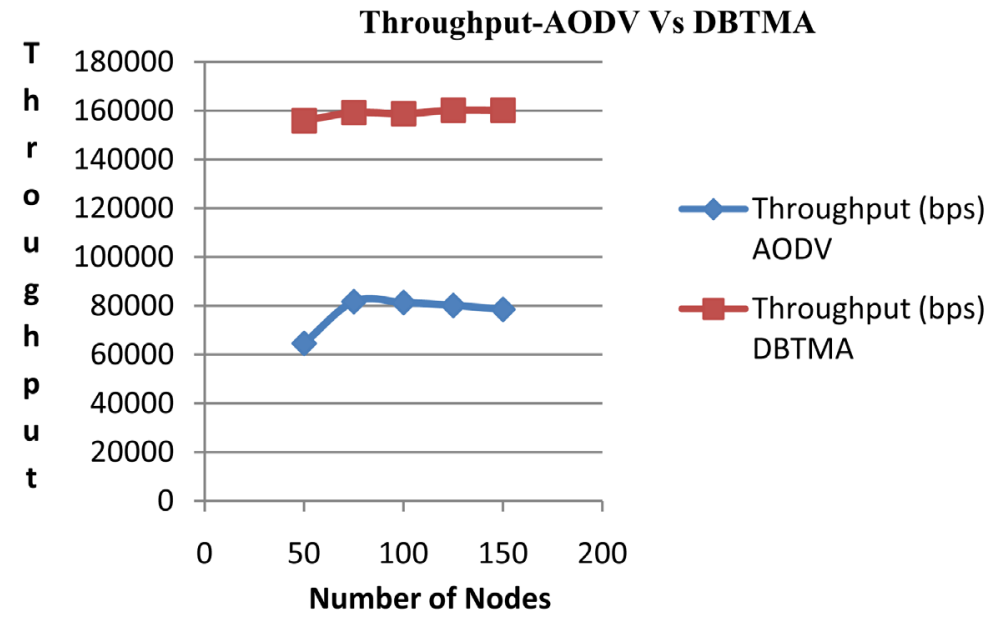

Figure 9. Data packets wise throughput-AODV vs DBTMA. 
Table 2. Hops per route in route discovery process.

\begin{tabular}{ccc}
\hline Destination & Next Hop & Cost \\
\hline A & $\times$ & $\times$ \\
B & B & 4 \\
C & E & 2 \\
D & E & 4 \\
E & E & 1 \\
\hline
\end{tabular}

Table 3. Number of Hops per route in AODV vs DBTMA.

\begin{tabular}{ccc}
\hline \multirow{2}{*}{ No. of Hops per Route } & \multicolumn{2}{c}{ Time (ms) } \\
\cline { 2 - 3 } & AODV & DBTMA \\
\hline 180 & 1.58 & 1.56 \\
576 & 1.52 & 1.51 \\
774 & 1.51 & 1.5 \\
972 & 1.5 & 1.49 \\
1170 & 1.5 & 1.47 \\
1368 & 1.5 & 1.47 \\
1566 & 1.5 & 1.47 \\
1784 & 1.5 & 1.47 \\
& 1.5 & 1.47
\end{tabular}

Table 4. Average end-end delay-AODV vs DBTMA.

\begin{tabular}{ccc}
\hline \multirow{2}{*}{ Number of nodes } & \multicolumn{2}{c}{ Delay (ms) } \\
\cline { 2 - 3 } & AODV & DBTMA \\
\hline 50 & 1.0992 & 0.4992 \\
15 & 0.1324 & 0.1946 \\
125 & 0.3814 & 0.1626 \\
150 & 0.2927 & 0.038 \\
\hline
\end{tabular}

Table 5. Data packets vise throughput-AODV vs DBTMA.

\begin{tabular}{ccc}
\hline \multirow{2}{*}{ Number of nodes } & \multicolumn{2}{c}{ Throughput (bps) } \\
\cline { 2 - 3 } & AODV & DBTMA \\
\hline 50 & 64542.4 & 155850 \\
75 & 81664 & 159200 \\
100 & 81292.8 & 158750 \\
125 & 80132.8 & 160200 \\
150 & 78499.2 & 160200 \\
\hline
\end{tabular}




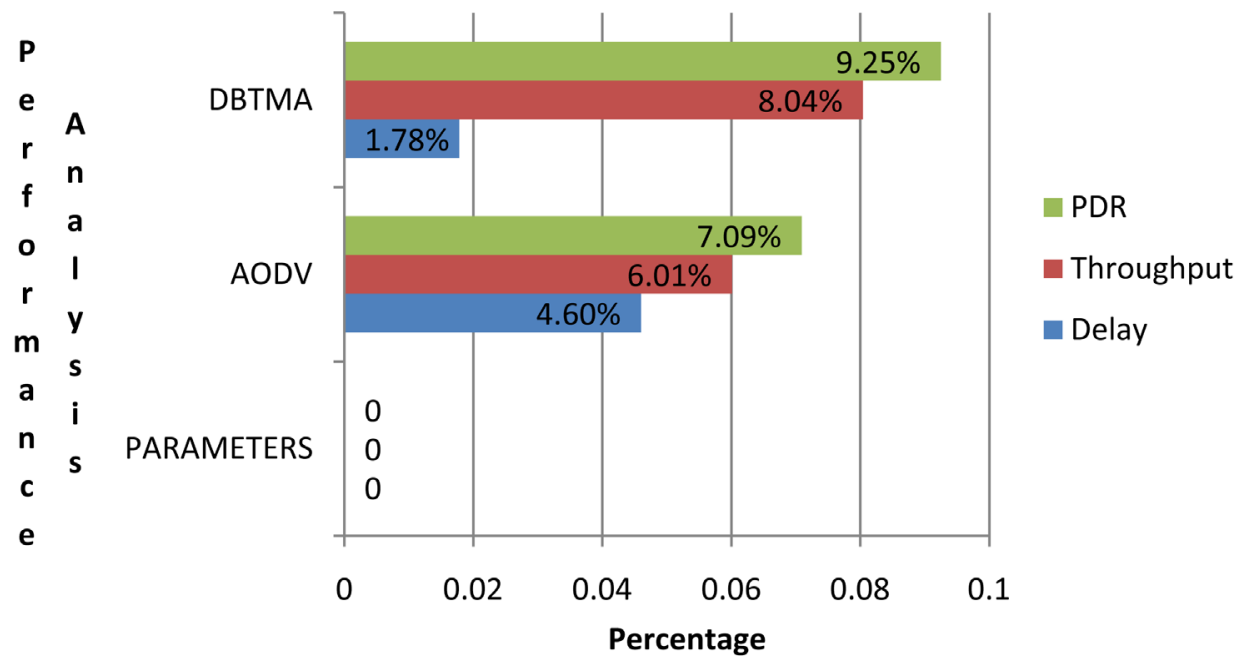

Figure 10. Comparison between AODV and DBTMA.

Table 6. Overall performance DBTMA on AODV.

\begin{tabular}{cccc}
\hline & PARAMETERS & AODV & DBTMA \\
\hline 1 & DELAY & $4.6 \%$ & $1.78 \%$ \\
2 & THROUGHPUT & $6.01 \%$ & $8.04 \%$ \\
3 & PDR & $7.09 \%$ & $9.25 \%$ \\
\hline
\end{tabular}

\section{Conclusion}

The results show that the impact of DBTMA on AODV has lower delay, an increase in throughput and efficiency in route discovery time, and number of hops per route. Thus, through implementation of On-Demand routing protocols like AODV along with the MAC protocol DBTMA can provide increase in its overall performance (shown in $\mathrm{Ta}$ ble 6). Since, any routing protocol cannot protect the data packet without the impact of a MAC protocol [4], and here it is DBTMA. The reason for the fusion scheme which has better performance is that delay avoidance using DBTMA theory increases the observation accuracy by combining the observations from networks. The performance analyses of AODV and DBTMA are shown in the Figure 10.

\section{References}

[1] Gupta, A.K., Sadawarti, H. and Verma, A.K. (2010) Performance Analysis of AODV, DSR and TORA Routing Protocols. IACSIT International Journal of Engineering and Technology, 2, 226-231.

[2] Youssef, M.A., Vasan, A. and Miller, R.E. (2002) Specification and Analysis of the DCF and PCF Protocols in the 802.11 Standard Using Systems of Communicating Machines. 10th IEEE International Conference on Network Protocols, Paris, 12-15 November 2002, 46-57. http://dx.doi.org/10.1109/icnp.2002.1181394

[3] IEEE P802.11e/D13.0 (2005) Part 11: Wireless Medium Access Control (MAC) and Physical Layer (PHY) Specifications: Amendment. 
[4] ANSI/IEEE Std 802.11 (1999) Part 11: Wireless LAN Medium Access Control (MAC) and Physical Layer (PHY) Specifications.

[5] Medium Access Control (MAC) Quality of Service (QoS) Enhancements, September 2004.

[6] Haas, Z.J. and Deng, J. (2002) Dual Busy Tone Multiple Access (DBTMA)-A Multiple Access Control Scheme for Ad Hoc Networks. IEEE Transactions on Communications, 50, 975-985. http://dx.doi.org/10.1109/TCOMM.2002.1010617

[7] Grilo, A. and Nunes, M. (2002) Performance Evaluation of IEEE 802.11e. The 13th IEEE International Symposium on Personal, Indoor and Mobile Radio Communications, Lisboa, 18 September 2002, 511-517.

[8] Choi, S., Del Prado, J., Shankar, S. and Mangold, S. (2005) IEEE 802.11e Contention-Based Channel Access (EDCF) Performance Evaluation. Proceedings of the IEEE International Conference on Communications 2003, 2, 1151-1156.

\section{Submit or recommend next manuscript to SCIRP and we will provide best service} for you:

Accepting pre-submission inquiries through Email, Facebook, LinkedIn, Twitter, etc. A wide selection of journals (inclusive of 9 subjects, more than 200 journals)

Providing 24-hour high-quality service

User-friendly online submission system

Fair and swift peer-review system

Efficient typesetting and proofreading procedure

Display of the result of downloads and visits, as well as the number of cited articles Maximum dissemination of your research work

Submit your manuscript at: http://papersubmission.scirp.org/ 